\title{
Molecular characterization of a chromosomal region involved in the oxidation of acetyl- CoA to glyoxylate in the isocitrate-lyase- negative methylotroph Methylobacterium extorquens AM1
}

\author{
Ludmila V. Chistoserdova and Mary E. Lidstrom
}

Keck Laboratories

138-78, California Institute of Technology, Pasadena, CA 91125, USA
Author for correspondence: Mary E. Lidstrom. Tel: +1 818395 2132. Fax: +1 8183952940. e-mail: LIDSTROM@CCO.CALTECH.EDU

A region on the Methylobacterium extorquens AM1 chromosome previously shown to complement a chemically induced mutant (PCT48) unable to convert acetyl-CoA into glyoxylate was characterized in detail in order to identify the gene(s) involved in the unknown pathway for acetyl-CoA oxidation. Six complete and two partial ORFs were identified by sequencing. Sequence comparisons suggested these might code for, respectively, a dehydrogenase of unknown specificity, a polypeptide of at least $15 \mathrm{kDa}$ with unknown function, a coenzyme- $B_{12}$-linked mutase, a catalase, an alcohol dehydrogenase (ADH) of unknown function, a polypeptide of 28 kDa, a ketol-acid reductoisomerase and a propionyl-CoA carboxylase (PCC). Insertion mutations were introduced into each ORF in order to determine their involvement in $\mathbf{C}_{1}$ and $\mathbf{C}_{2}$ metabolism. Mutations in three genes, encoding the mutase, ADH and PCC, resulted in a phenotype characteristic of mutants unable to oxidize acetyl-CoA, i.e. they were $C_{1}-$ and $C_{2}$-negative and their growth on these compounds was restored by the addition of glycolate or glyoxylate. Mutants in the genes thought to encode catalase and PCC were found to be deficient in the corresponding enzyme activity, confirming the identity of these genes, while physiological substrates for the mutase and ADH remain unidentified. This work, in which three new genes necessary for conversion of acetyl-CoA into glyoxylate were identified, is an intermediary step on the way to the solution of the unknown pathway for acetyl-CoA oxidation in isocitrate-lyase-negative methylotrophs.

Keywords: Metbylobacterium extorquens, isocitrate-lyase-negative methylotrophs, serine cycle, acetyl-CoA oxidation, $\mathrm{C}_{1}$ and $\mathrm{C}_{2}$ metabolism

\section{INTRODUCTION}

Metbylobacterium extorquens AM1 is a pink-pigmented serine cycle methylotroph able to grow on methanol and methylamine, and also on a variety of multicarbon substrates including ethanol, ethylamine, lactate, pyruvate, malonate, $\beta$-hydroxybutyrate and succinate (Peel \& Quayle, 1961; Tsuji et al., 1990). For more than two

Abbreviations: $A D H$, alcohol dehydrogenase; HPR, hydroxypyruvate reductase; Km, kanamycin; MCM, methylmalonyl-CoA mutase; PCC, propionyl-CoA carboxylase; Tc, tetracycline.

The GenBank accession numbers for the nucleotide sequences of the 8988 bp Hind III-ECORI fragment and the 285 bp EcoRl-HindIII fragment reported in this paper are $L 48340$ and $L 48243$, respectively. decades, this organism has been used as a model for studying both the biochemistry and the genetics of the serine cycle utilized for assimilation of $\mathrm{C}_{1}$ compounds at the level of formaldehyde (Salem \& Quayle, 1971; Dunstan et al., 1972a, b; Dunstan \& Anthony, 1973; Anthony, 1982; Chistoserdova \& Lidstrom, 1992, $1994 a-c)$. In the serine cycle, formaldehyde in the form of methylenetetrahydrofolate condenses with glycine to produce serine, which then undergoes transamination with glyoxylate, producing hydroxypyruvate, which in turn is reduced to D-glycerate. The latter is phosphorylated to phosphoglycerate, then transformed to phosphoenol pyruvate, which is carboxylated to produce malate and then malyl-CoA. This is then cleaved to glyoxylate and acetyl-CoA (Anthony, 1982). In the serine 
methylotrophs which lack isocitrate lyase $\left(\mathrm{Icl}^{-}\right)$, such as $M$. extorquens AM1, the second molecule of glyoxylate must be regenerated from acetyl-CoA, but the biochemical pathway leading to such a conversion remains unknown (Anthony, 1982). Studies of the fate of carbon atoms originating from ethanol, acetate or methanol have indicated that a common pathway must operate that leads from acetyl-CoA to glyoxylate during assimilation of $\mathrm{C}_{1}$ and $\mathrm{C}_{2}$ compounds, and also multicarbon compounds like malonate and $\beta$-hydroxybutyrate (Salem \& Quayle, 1971; Dunstan et al., 1972a, b; Dunstan \& Anthony, 1973).

The pathway of $\mathrm{C}_{2}$ assimilation also remains unknown in other bacterial groups (Dawes \& Sutherland, 1994). Elucidation of this pathway in methylotrophic bacteria might bring about greater understanding of the fate of acetyl-CoA in other $\mathrm{Icl}^{-}$organisms.

In $M$. extorquens AM1, three chromosomal fragments have been identified carrying genes encoding enzymes involved in assimilation of both $\mathrm{C}_{1}$ and $\mathrm{C}_{2}$ compounds and probably operating in the unknown pathway for glyoxylate regeneration. One of these, orf4, encodes a protein of unknown function and is located in a large cluster of $\mathrm{C}_{1}$ genes that includes a number of serine cycle genes (Chistoserdova \& Lidstrom, 1994c). Another gene, gly A, is located on a separate chromosomal fragment and encodes serine hydroxymethyltransferase. This enzyme apparently plays a dual role in $M$. extorquens AM1, firstly in the serine cycle and secondly in the oxidation of acetylCoA (Chistoserdova \& Lidstrom, 1994b). The third chromosomal fragment not linked to the two fragments mentioned above has been shown to complement a number of chemically induced mutants such as PCT48 that are unable to grow on $\mathrm{C}_{1}$ and $\mathrm{C}_{2}$ compounds and are complemented by the addition of glycolate or glyoxylate (Stone \& Goodwin, 1989; Smith \& Goodwin, 1992; Smith et al, 1995). PCT48 was shown to contain a deletion of $3.3 \mathrm{~kb}$ in this region (Smith \& Goodwin, 1992). The goal of the present study was to characterize this last region in more detail.

\section{MATERIALS AND METHODS}

Bacterial strains, plasmids and growth conditions. Escberichia coli strains DH5 $\alpha$ (Bethesda Research Laboratories) and S17-1 (Simon et al., 1983) were used in this study. They were grown in LB medium in the presence of appropriate antibiotics as described by Maniatis et al. (1982). M. extorquens AM1 was grown in the minimal medium described previously (Harder et al., 1973). Succinate $(20 \mathrm{mM})$, methanol $(100 \mathrm{mM})$, methylamine $(20 \mathrm{mM})$, ethylamine $(20 \mathrm{mM})$, formate $(40 \mathrm{mM})$, pyruvate $(40 \mathrm{mM})$ or $\beta$-hydroxybutyrate $(20 \mathrm{mM})$ were used as substrates. Methanol induction of mutants was carried out as described by Dunstan et al. (1972b). The following antibiotic concentrations were used for $M$. extorquens AM1: tetracycline (Tc), $10 \mu \mathrm{g} \mathrm{ml}^{-1}$; kanamycin $(\mathrm{Km}), 100 \mu \mathrm{g} \mathrm{ml}^{-1}$; and rifamycin, $50 \mu \mathrm{g} \mathrm{ml}^{-1}$. The growth responses of mutants were tested on plates containing the substrates listed above in the presence or absence of supplements of glyoxylate $(5 \mathrm{mM})$ or glycolate $(20 \mathrm{mM})$.
DNA-DNA hybridizations. These were carried out with dried agarose gels as described by Meinkoth \& Wahl (1984) at $68^{\circ} \mathrm{C}$. SSC (0.15 M NaCl, $0.015 \mathrm{M}$ sodium citrate) was used at $6 \times$ concentration for hybridizations and $0.5 \times$ concentration for washes.

DNA manipulations. Plasmid isolation, E. coli transformation, restriction enzyme digestion, ligation, blunting ends with $\mathrm{T} 4$ DNA polymerase, or filling in ends with Klenow enzyme were carried out as described by Maniatis et al. (1982). The chromosomal DNA of $M$. extorquens AM1 was isolated by the procedure of Saito \& Miura (1963).

DNA sequencing. The DNA sequence of both strands was determined with an Applied Biosystems automated sequencer by the Caltech Sequencing Facility.

Computer analysis. Translation and analyses of DNA and DNA-derived polypeptide sequences were carried out using PC/Gene (Genofit, Geneva) or Genetic Computer Group (Wisconsin) programs.

Enzyme assays. Enzyme activities were determined in $M$. extorquens AM1 crude extracts prepared as described previously (Chistoserdova \& Lidstrom, 1992). All measurements were done at room temperature $\left(26^{\circ} \mathrm{C}\right)$ in a total volume of $1 \mathrm{ml}$. Hydroxypyruvate reductase (HPR) was assayed as described by Chistoserdova \& Lidstrom (1991). Catalase was assayed by following $A_{240}$ (Beers \& Seizer, 1952). Propionyl-CoA carboxylase (PCC) was assayed by following the appearance of ADP. The reaction mixture contained $50 \mathrm{mM}$ Tris $/ \mathrm{HCl}, \mathrm{pH}$ 7.5;3 mM ATP; $10 \mathrm{mM} \mathrm{MgCl}_{2} ; 3 \mathrm{mM}$ propionyl-CoA;0.2 mM $\mathrm{NADH} ; 3 \mathrm{mM}$ phosphoenol pyruvate (PEP); 20 units pyruvate kinase $(\mathrm{PK}) ; 7$ units lactate dehydrogenase $(\mathrm{LDH})$ and crude extract containing $0 \cdot 1-0.3 \mathrm{mg}$ protein. MethylmalonylCoA mutase (MCM) was measured by disappearance of ADP in a discontinuous assay coupling MCM and PCC reactions. For this assay, PCC was overexpressed by introduction of cloned $\operatorname{pcc} A$ (see Results). The reaction mixture contained, in $0.5 \mathrm{ml}$ $50 \mathrm{mM}$ Tris/HCl, pH 7.5: 0.5 mM ADP, $5 \mathrm{mM} \mathrm{MgCl}_{2}, 3 \mathrm{mM}$ succinyl-CoA, and crude extract containing $0.1-0.3 \mathrm{mg}$ protein. The reaction was allowed to proceed for 5-10 $\mathrm{min}$, after which the remaining ADP was measured in $\mathrm{LDH}$ reaction by the addition of $0.5 \mathrm{ml}$ of the following mixture: $50 \mathrm{mM}$ Tris $/ \mathrm{HCl}$, $\mathrm{pH} 7.5 ; 3 \mathrm{mM}$ PEP; $0.2 \mathrm{mM}$ NADH; 20 units PK; and 7 units LDH. Spectrophotometric methods (Kalb \& Bernlohr, 1977; Whitaker \& Granum, 1980) were used for protein determination.

Isoelectrofocusing. Crude cell extracts were isoelectrofocused in a $\mathrm{pH}$ range of 3-9 or 4-6.5 using PhastSystem as described by the manufacturer (Pharmacia-Biotech). For alcohol dehydrogenase $(\mathrm{ADH})$ assays, gels were stained using a reaction mixture consisting of a buffer (potassium phosphate, $\mathrm{pH} 7 \cdot 0$; Tris $/ \mathrm{HCl}$, $\mathrm{pH} 7 \cdot 5-8 \cdot 0$; or pyrophosphate, $\mathrm{pH} 9 \cdot 0$ ) plus $1 \mathrm{mM}$ NAD or NADP, $1 \mathrm{mM}$ nitro blue tetrazolium, $0.5 \mathrm{mM}$ phenazine methosulphate and 1-10 $\mathrm{mM}$ substrate (ethanol, 2-propanol, glycerol, acetaldehyde, propionaldehyde, glycolaldehyde, glycolate or glycerate). Catalase was visualized by incubating gels in a buffered $(\mathrm{pH} 7 \cdot 0) 3 \%(\mathrm{v} / \mathrm{v})$ solution of hydrogen peroxide and noting the appearance of bubbles.

Matings. Triparental or biparental matings between E. coli and $M$. extorquens AM1 were performed overnight on nutrient agar at $30^{\circ} \mathrm{C}$. Cells were then washed with sterile medium and plated on selective medium at appropriate dilutions. In triparental matings, pRK2013 (Ditta et al., 1985) was used as a helper plasmid. Rifamycin was used for E. coli counter-selection. 
Acetyl-CoA oxidation genes in a methylotroph

\section{RESULTS}

\section{Identification of the gene impaired in mutant PCT48 and sequence of adjacent DNA regions}

In $M$. extorquens AM1, a $13.5 \mathrm{~kb}$ HindIII fragment was shown to be responsible for complementation of PCT48, a mutant impaired in the conversion of acetyl-CoA to glyoxylate (Stone \& Goodwin, 1989). This fragment was subcloned from a cosmid pSS48.11 (supplied by P. Goodwin) into pUC19 (Pharmacia) and sequenced. A $4.2 \mathrm{~kb}$ EcoRI fragment within this region has been sequenced elsewhere, and analysis of these data has shown that the lesion in the mutant PCT48 was in a gene (mea $A$ ) highly similar to genes from various sources encoding a coenzyme- B $_{12}$-linked enzyme, MCM (Smith et al., 1995). We have independently sequenced this region, and have confirmed that finding. Here we report the sequence of regions flanking mea $A$. The physical map of the $13.5 \mathrm{~kb}$ chromosomal fragment employed in this study is shown in Fig. 1. Six complete and two partial ORFs found in the region are indicated. Fig. 2 shows the sequence of the $285 \mathrm{bp}$ HindIII-EcoRI fragment at the left (Fig. 1a) and the 8988 bp EcoRI-HindIII fragment at the right (Fig. 1b) of the $13.5 \mathrm{~kb}$ fragment shown in Fig. 1. The sequence of the middle $4.2 \mathrm{~kb}$ EcoRI fragment will be published elsewhere (L. M. Smith and others, unpublished results). Analysis of the sequence revealed six complete and two incomplete ORFs. Reading from left to right on Fig. 1, the first (partial) ORF potentially encodes the C-terminal $25060 \mathrm{Da}$ of a larger polypeptide, the second could encode a polypeptide of at least $15 \mathrm{kDa}$ (we have not completed the double-stranded sequence of this gene) and the third encodes MeaA (78293 Da) (Smith et al., 1995). The fourth ORF is potentially able to encode a protein of $55550 \mathrm{Da}$. A fifth ORF able to encode a polypeptide of $47529 \mathrm{Da}$ begins $82 \mathrm{bp}$ from the fourth. The next $894 \mathrm{bp}$ do not seem to contain any ORFs and separate the fifth ORF from a divergently transcribed ORF, potentially able to encode a polypeptide of $28937 \mathrm{Da}$. This sixth ORF is separated by $167 \mathrm{bp}$ from a seventh, transcribed in the same direction, potentially able to encode a polypeptide of $36879 \mathrm{Da}$. The eighth (partial) ORF in the region begins beyond the described fragment and could encode the Cterminal $55199 \mathrm{Da}$ of a larger polypeptide. A region of $1723 \mathrm{bp}$ separating the seventh ORF from the eighth one seems to be a non-coding region. A few inverted repeat sequences having the potential to form a stable stem-looptype structure were found in the described region [hairpins with free energy exceeding $-14 \mathrm{kcal}$ (approx. $-58.5 \mathrm{~kJ}$ ) are underlined in Fig. 2], which could serve as terminators. The structures with the highest calculated free energies are found immediately downstream of the fourth ORF [calculated $\Delta G\left(25 \cdot 5^{\circ} \mathrm{C}\right)-35 \cdot 8 \mathrm{kcal}$ (approx. $-149.6 \mathrm{~kJ})$ ], the eighth ORF [calculated $\Delta G\left(25.5^{\circ} \mathrm{C}\right)$ $-24.6 \mathrm{kcal}$ (approx. $-102.8 \mathrm{~kJ}$ )] and the seventh ORF [calculated $\Delta \mathrm{G}\left(25.5^{\circ} \mathrm{C}\right)-20.8 \mathrm{kcal}$ (approx. $\left.-86.9 \mathrm{~kJ}\right)$ ].

\section{Amino acid sequence comparisons}

The amino acid sequences deduced from complete and partial ORFs found in the region under study were



Fig. 1. Physical and genetic map of the $13.5 \mathrm{~kb}$ Hindlil fragment of the $M$. extorquens AM1 chromosome able to complement mutant PCT48. Arrows show the direction of transcription, and asterisks show sites of the $\mathrm{Km}^{r}$ gene insertions with corresponding mutation names below.

compared against the protein databank. The first (partial) ORF showed similarity with a number of dehydrogenases, including 3-oxoacyl-[acyl-carrier-protein] reductase (about $35 \%$ identity; Klein et al, 1992; Slabas et al., 1992), glucose 1-dehydrogenase (about 32\% identity; Lampel et al., 1986; Heilmann et al., 1988), acetoin (diacetyl) reductase (about $25 \%$ identity; Blomqvist et al., 1993) and acetoacetyl-CoA reductase (about $23 \%$ identity; Peoples \& Sinskey, 1989). This gene was designated $x d h$, for unknown dehydrogenase. No significant similarity to known protein sequences was found for the second ORF, which was designated orf1. The polypeptide deduced from the third ORF showed high identity with subunits of MCM from both prokaryotic and eukaryotic sources (about 35\% ; Francalanci et al., 1986; Jansen et al., 1989; Wilkemeyer et al., 1990), and has been designated mea $A$ (Smith et al., 1995). The next ORF, transcribed divergently from mea $A$, was shown to encode a polypeptide highly similar to catalase subunits from various sources (Murthy et al., 1981; Schroeder et al., 1982; Bell et al., 1986; Furuta et al., 1986; Orr et al., 1990; Bol et al., 1991) with identities ranging from 40 to $57 \%$, and was designated kat $A$. The gene downstream of kat $A$ encodes a polypeptide with similarity to NAD-dependent ADHs belonging to different classes having specificity for shortchain alcohols (24-34\% identity; Russell et al., 1983; Russell \& Hall, 1983; Young \& Pilgrim, 1985; Gwynne et al., 1987; Saliola et al., 1990) and was designated adh $A$. The polypeptide translated from the next ORF, transcribed divergently from adh $A$ did not reveal strong identity to known proteins and was designated orf2. The putative product from the seventh ORF was shown to bear high similarity to $i l v C$ gene products from bacteria and yeasts, with identities ranging from 32 to $54 \%$ (Daniels et al., 1992; Godon et al., 1992), and was designated $i l v C$. The last (partial) ORF in the region encodes a polypeptide highly similar to the $\beta$-subunit of mammalian PCC (about $60 \%$ identity; Kraus et al., 1986; Lamhonwah et al., 1986), and was designated $p c c A$. 
(a) 5' AAGCTTGACCTAAGGTCGCGGTGCGTCACCGGTGCGTCGAAGGGGATCGGCGCCGCCATCGCAAAAGCATTGGCGAAGGATGGCGCGGCCGTCGTCGTCAACTACGCATCGAGCAAGGCC 5' GGCGCGGATGCCGTCGTCGAAGCCATCACGGCCGCCGGCGGCAAGGCCATCGCGGTCCAGGCCGATGTCTCGCAGGCCCGTGCAGGCGCGGGGCTTGGTTGAGGCCGCGGTGCAGCAATTC $G$ A D A V V E A I T A A G G K A I A V Q A D V S Q A V Q A R G L V E A A V Q Q F 5' GGCCGGCTCGACGTGCTGGTGAACAATTCCGGCGTCTACGAATTC $G \quad R$ L $Y$ L $V$ N N S G $V$ Y E

(b) 5. GAATTCGCTCACCGCCGGCTCGCGTGGGCCGGTTCTGATGCAGGACTACCACCTCATCGAGAAGATGGCCCACTTCAACCGGGAGCGGATCCCGGAGCGGGTGGTGCACGCCAAGGGCTA



5. CGGCCCCTACGGCACGTTCCGGCTCACCAGAAGCCTGTCCGAGTTCACCCGTGCCAAGGTGCTGACCGAGGTCGGCAAGGACGTGCCGATGGTGGCGCGCTTCTCCACCGTCGGCGGCGA



5. GTCCGGTTCGGCCGACACCGCCCGCGACCCGCGCGGCTTCGCCCTCAAGTTCTACACCGAGGAGGGCAACTGGGATCTCGTCGSCAACAACACCCCGATCTTCTTCGTGCGCGACGCGAT

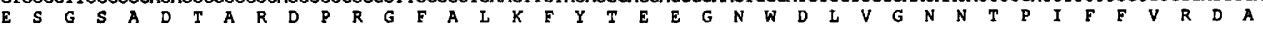

5. CAAGTTCTCCGACTTCATCCGCACCCAGAAGCGCGATCCGCGCACCCACCTCAAGCCGCATTGGCGCCGCTGGGACTTCTGGAGCCTGTCGCCGGAGGCGATCCATCAGGTCATGTTCCT

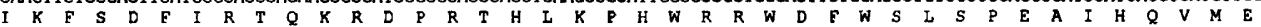

5. GTACTCGGATCGCGGCACGCCGAAATCGGCOCGCTTCATGAACGGCTACGGCTCGCACACCTTCTCGCTCTGGAACGACCGGGGCGAGCGCCACTGGGTGAAGTTCCACTTCCACACCAA



5. GCAGGGCATCCAGAACTTCTCGGCCGACGAAGCGACGAGGTAGCGGGCAAGGATCCGGACTACTCCTCCGCCGACCTCTCGACTGCCATCGAGCAGGGCGACTTCCCGAAATGGAAGGT $\begin{array}{lllllllllllllllllllllllllllllllllllllllll}K & Q & G & I & Q & N & F & S & A & D & E & A & D & E & V & A & G & K & D & P & D & Y & S & S & A & D & \text { L } & S & T & A & I & E & Q & G & D & F & P & K & W & K\end{array}$

5' CVTCCGTGCAGCTCATGCCGGAATTGGACGCCGACACCTACCGCATCAACCCGTTCGACCTGACCAAGGTCTGGCCGCACGGCGACTATCCGCTGATCGAGATTGGCGAGATGGAGCTCAA

5. CCGCAATCCCGAGAACTACTTCGCCGAGATCGAGCAATCTGCCTTCGAGCCCTCGAACGTGGTGCCCGGCATCGGTTTCTCGCCGCACAAGATGCTGCAGAACCGGGTGCTGTCCTATGC N R N P E N Y F A I E S A F E S N V V P G I G F S P H K M L Q N R V L S Y

5. CGACGCCCACCGCTACCGGCTCGGCGTGAACTACCAGCAGATCCCGGTGAACCAGGCGAAAAACGCTGACGTTCAGACCTACCATCGCGACGGCGCCATGCGCACCGACGGCAATCACGG A D A H R Y R L G Y N Y Q Q

5. CGCCCAGGTCGATTACGAGCCGAACTCCTTCGGCGGTCCGAAACAGGACCCGTCCTTCAGCGAGCCGCCACTGCGGATCCAGGGCGATGCTGGCCGCTACGGTTGGCCGGGCGACGACGA G 5' GGATCTCTACGGTCAGCCCAAGCTGTTCTGGACCAAGGTGCTGGATGAGGGCGGCCGCAAGCGGCTCGTCGAGAATATCGTCACCTCGATGGGCGACAGCCCGCGCCACATTCAGGAGCG 5. GATGATCGCGCACTGGTTCAAGGTGCACGAGGATTTCGGGCGCGGTGTGGCCCAGGGGCTCGGCATCGATACGGCACAGGCCGCCGCCGAATAGTCATCTCAACATTATTTTGAATACAG

5. GGGCATCGACGTTGGTCGGTGCCTCTTTTTTGTGCGGACGAGGCAAAGCCTTGCCTTTTAGACCACTTGGAAGGCTGGAAACATCGTGCTTATAGCGTCGGCCAAGGTCGCGCGGTTCGT

5. CACGCGCGTGCCAGAACCAACAGGGAGTGCCACGATGGCTGCAAGCGCAGCACCGGCCTGGACCGGGCAGACGCCGGAAGCCAAGGACCTTTACGAGCTCGGCGAGATCCCCCCGCTCGG

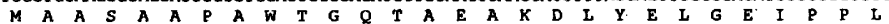

5. CCACGTGCCTGCCAAGATGTATGCCTGGGCGATCCGCCGCGAGCGCCACGGGCCGOCGGAGCAGTCGCACCAGCTCGAAGTGCTTCCCGTCTGGGAGATCGGCGACGACGAGGTGCTCGT

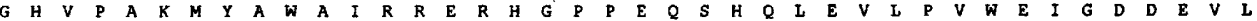

5. CTACGTCATGGCCGCGGGCGTGAACTACAACGGCGTTTGGGCCGGCCTGGGCGAGCCTATCTCGCCGTTCGACGTGCACAAGGGCGAGTACCACATCGCCGGCTCGGACGCGTCGGGTAT

1. CGTCTGGAAGGTGGCGCCAAGGTGAAGCGCTGGAAGGTCGGCGACGAGGTCATCGTCCATTGTAACCAGGACGACGGCGACGACGAGGAGTGCAACGGCGGCGATCCGATGTTCTCGCC

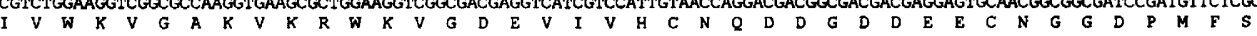

51 GACCCAGCGGATCTGGGGTTACGAGACCGGCGACGGTTCGTTCGCGCAGTTCTGCCGGGTGCAGTCGCGTCAGCTCATGGCCCGCCCCCAAGCACCTGACCTGGGAAGAGGCCGCCTGCTA

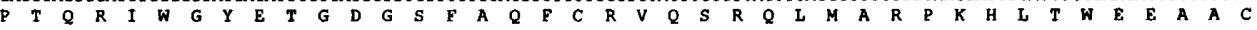

5. CACGCTGACGCTCGCCACCGCCTACCGCATGCTGTTCGGCCACGCGCCGCACACCGTGCGTCCGGGCCAGAACGTGCTGATCTGGGGCGCCTCCGGCGGCCTCGGCGTGTTCGGCGTCCA



5. GCTCTGCGCGGCCTCCGGCGCCAACGCCATCGCCGTGATCTCGGACGAGTCGAAGCGCGACTACGTGATGAGCCTCGGTGCGAAGGGCGTCATCAACCGCAAGGACTTCGACTGCTGGGG $\begin{array}{lllllllllllllllllllllllllllllllllllllllll}Q & \text { L } & C & A & A & S & G & A & N & A & I & A & V & I & S & D & E & S & K & R & D & Y & V & M & S & I & G & A & K & G & V & I & N & R & K & D & F & D & C & W\end{array}$

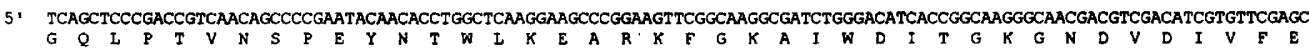

5. TCCCGGAGAGGCGACCTTCCCGGTCTCGACGCTGGTGGCCAAGCGCGGCGGCATGATCGTGTTCTGCGCCGGCACCACCGGCTTCAACATCACCTTCGACGCCCGGTACGTCTGGATGCG H P G E A T F P V S T L V A K R G G M I V F C A G

5. GCAGAAGCGCATCCAGGGCTCGCACTTCGCCCACCTCAAGCAGGCCTCGGCCGCCAACCAGTTCGTGATGGACCGGCGCGTCGATCCCTGCATGAGCGAGGTGTTCCCCTGGGACAAGAT $R Q K R$ I Q G S H F A H I K Q A S A A N Q F V M D R R V D P C M S E V F P W D K

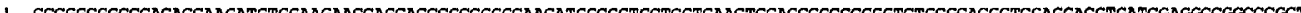



5. CAAGGCGATGTGAGCCAGTCCACGTGACACGAAGAAGCCCGCGTATCCCGGTGGATGCGCGGGCTTCTITCGTTGGGGGACGCGTTCAGCGCACCCGGTCGATCGCCCGCTGCGGTGTGC K A M

5! CGATCGTGGTGTGGAACGGCCGTTCACCGCCCCCCTTCTCGAAGGCGACGATGCTGTCGAAGACGTGGATCGCGCCGATGCTCCGGCCCGATCTCCTGCGCGCCCCCGGTCGTGATACCAAG

5. CGTGCATGTCGTCGATGAACCCCTTCGTCATCTCCAGGAACGTGCCCCGGCGGCGGTAGCCGCCCTCGTATCCCGCCAGTACGAGGTGTGCAGGTCTTCGGCGACGTAGAGACCGTCTGG

5. CGCCAGGAGCGGAAACAGCGTCTCGAAGGTGACGCGTTGGTGGCTCGCGACGTGGCTGCCGTCGTCGAGGACGACATCGACGCCGCCCATCTCCTCGACCACGGACCGAAGGAAAGCGGG

5. ATCGGCCTGCGAGCCGATACGGACCTGTGCGGCGCGACCGTCGAACACCGCGCAGCGCGGATCGACATCGACGCCGTAGAGAATGGCGCTCGGTCCGAAGAAACGACGCCAAAGGTCGAG

5. GGAGCCGCCCTCGTAGACGCCGATCTCGAGGAAGCGCAGCGGTCGCCCGTCCGTCCTGCCGTCGCGGAAGGGCGCGAACAGCCGGCTGTAGATCGGCAGGAAGTGGTTCCACTTGTTGAT

3. CCTCGGCGGGAGCATCTGCGGCTAGAGCTCCTTCGCGTCGCCAGCGGGCAGGCAGGACGGCAGCGCCTTCCCGCGCTTGTCGGCCGACATCTAGCCGTCCTTCACCAAGGTGAACAACTA

3. GTCCACCAGCGGCAGTATCGGCATCTTGTCGAAGCTCTTCCGCAGCAGCGGGGCGCAGAAATGGAGCCGGTCTAGCGCTATCTTCAGCAACCGGCCCGACAACGCTTTATTAGGGCGACT

3. AAACCGCTGCCTGAATTCACGGGCAGAACTAGACGCCTACTAACACGTCGCAAGCGAATTCTTTGCAGTAAGGCTCTACGCCGGGCAGCACGACAGGCTCCAGCGCCCGTGCTTCGTGCT



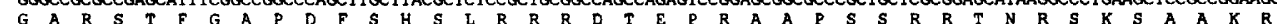

120

240

360

480

720

840

960

1080

1200

1320

1440

1560

1680

1800

1920

2040

2160

2280

2400

2520

2640

2760

2880

3000

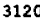

3240

3360

3480

3600

3720

3840

3960

Fig. 2. For legend see opposite. 
3. ACCGCTGCGCGCGGAACAGGAGCAGCGACTACTGCAGCGGCCGTTCTAGCTCGGCGAAGGTCCGGCGCTCGCCGCAGAGGGCCAGCCCGCCCCCTGCATGAACAAGACGAGCTCGAAGAA

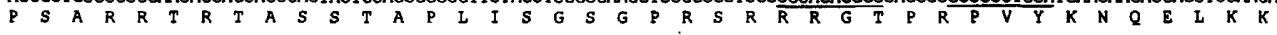
31. CATGAATACCTACATCGCGCCCGTCAACTAGGTCTAGAACCAGTGTAGCTGGGCGTGCTCTATGAAGCGGAGGCACATGAGCCGGTAGTCGCCTAGCAGCGCGTCGTCGCTCCGCACTGG 1200

3. GACCTGCGCCTACGCCACGCCGCAGAGCTTCAGCTATTTGTCCTCCGGCTAGAACCGGACGGGGAGCTACAACGGGTAGCTCATCTTGTAGGGCAACGGCAGCTTCGGCCCCTTCTGGTC 320

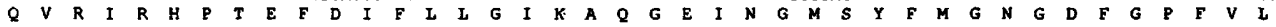

3. GCAGCTCGGCAGCTGGTGGAATGCTTCGGCCAGCCCGGCGGGAACATCCTCTGCCAGCCGTACGGCAGCCCTAGTTGCCACCTCTCCTTCTTGTACAGCGCGCTCCGCTACTTCCGCCG 4440

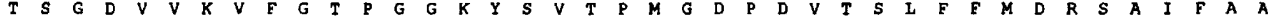

31 GAGCAGCCCAACTTAGCAGAGCACTTGGGTTAGGAGCAGCTCCGCATGCCGCTCGAACCAGCACGGCTTAAGGAGGACCTCCCGCGCCACGCGGAGGCTCATCTCGCTACGGTATACGCA 4560 E D P Q I T

3. GAGGAGGCCCCGGCTAACGAAGCCCGGAGGACTCCCTCGGCAAATTCCGTTTCCGCGTTCCCGCGTTTGCOCCGGCGCTTTTTTCCGOCCAGTGCGAGCGCGCTTGGCCGGAGTCCGTGGTG

3. TCGCCTCCGGGCTCGCTTCGGGCCGGAGGTTCAGGCTTTTCTAGTCAAGAACCAGAACAGCTGGTCCCGGAAGAGCCTCTAGGTGCCGTAGTACGGTGCCTCGAACCGCGGCTGAAGGAGC * $K$ T K D V L A K E S I W P M M G R L K A G V E E I

31 TAGCCCACCCGCCGCTCGAACCGCGCCCACCGGAACTTGCTCCAGACCGGCTGGAACAAGAGGTCGTAGGTCAACGCCCACTTCTACGGGCTGACCTACAGTAACTCCTGCGCGAAGTAG

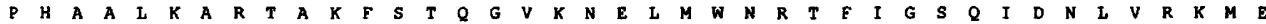
3. AGCCGGACCAGAGGCCGCACTGCTACGCGCCCGGCCACTGCATGAGCGGCATGAGCCGCCACAAGCTCTAGCTCATCAAGTACAACCGCTACGGCGGGAGCATCTACTCTAGCTGCTAG

3. TCGAAGTGGAGCACGTCCGTGAGCTTTATCCGGTAGAGCCCCCGCATCGGGCGGAGCTGGTCGCAGAGCTTCGGCCGGACTAGTCAAGCTGCTCCGGCGGCGTCTCGTGCCGGACGAGT

3. GGCTTGTCCAGCCAGAGCGTGAGAAGGAACTTCCACCAGAGCTACTACGGCCGCGCCCGGCGGCGGTAACCGGCTCCGCATCCTCTCCGGCTCCAGCACCGGCAACGGGCTCCGCAGGACC

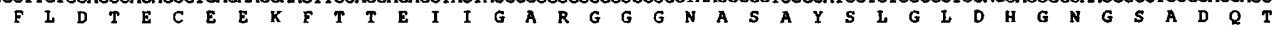

31 CACTACCTCTAGTCCCAGCCGTGCGGCGGCGGGAACTCCATGAGCGGCGCGTGCCACACCGGCCACGGGAAGCCCCGGTTGGTACTCGTGCAGCTCCAGGAACGCGCCGAGCTAGTCCAAC



3. TTCACGTGCAACTCCGGCACCCGCTTGTCCTCGCGCCGCGGGACGAAGTACAAGCCGAGGTCGCTGAGGAACATCTACAGCGGGACGTCGAGCAGGCCCCACTCGTAGTAGTGGTGCAGC

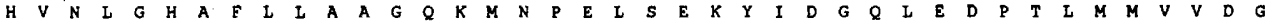

3. GGGTGAACCGCCGCAGCCGCTGCAAGTAGTGGAACTTCGGGAGCACGAGTCGGAAGAACCGCCACCGCCTGGGGAGCGCGTCCCGCTACTGCTACAGGAACTGCGGGCTTAGCGCGTCC 3. AAGTCGCGCACTCGTACCGGGACCCTCGGCATCGGCTGCTACTGCTGCAAGAACGGGAACTAGTCCAAGTCCAGCCGTAGCGCTAGCATTATCTGCGCGTACCACCACAGGAGAGAGCAC I A H A H G Q

3. AGTCACCAAAGCCAAGTCCCAAAGCGGGCGGGCATCTCCCGGTCCTTGACGAGCTGCCGTGCCCGGCGCAGCGAGGCCTAGAGCCGCCGCACCTCCAGCTCGCGCAGCGGCTCTTCTTCA

3. GCCTAGACTTATAGCGCCGGCTGGTCTGGTTTCTTCCAGGCCTTCCGGCGGAGCGACAGGAGCTTACGCTCGTCCGGCCGGCGCGCCGGGCGAAGCTCGTGGCCGAAGTGGGCGAGCGGC

31 TACCGCTTCGCCGGTAACCGGTCGTGCTACGGTGGCTCTAACGAGAAAGCCCTGGGGCGTAGCCGCTACCGCTATGCCAACTCGCGCTGGCTCCAGCCCGACCGTTACTGGCGTTCGGTC

31 GAGAGCCGCTTCAGCTCGTCCAAGAACGCGTCCCGCTGCCGTAGGTCGGGCGAGTGCAGCATCGCCGGCCGTACCTGGGCGCTCCGGACGGTCGCCTGGCACCGCCAGTCGTCTGGCAGC

3. GCCAGCGGCTTGGTCGCTATCTCGCAGAGGAAGCTCGTCGAGCGGGCCGCGCGGTGACGCAGGTACCACTCGCCTAGTGCCGGTAGCTGGTAGTCGCGGTTGCGCCGTAGGTCGTGGCGG

3. ACGACCGCGCCGCACCCCGCAGAGCCGGCTTTCGAGCAGCCACTACTCCCCCGAGCCGACGTACTAGGCCAACGACCTGTCATGGCATGGCATGCCATGCGAGCAGATTGGCTCGGTCGGC

3. GGGCCCTGTTCGCCCTACCAGCTCTAGCAGGCTAGGGCACGTGGCAGTGCAAAGACAGCGGCGGAACGACTTGGGCGAACGGCTCGCGGGTCGGAGCCGCATATGCCGGGAGGCAGTGGT

3. GTCGCTGCGGGCACAGCGGGTGGGCGCCGCCGT GCAGCCCACAAACCCACGCATCGAGTCGCCCTCTCGTAAGCGAAGTGTGCGCTTCCCAGT GTCCAAGCGAGGGACAGCGCGGGTGGT

31 GGCGGGGAGGTGCCGTCAAGCCAGCAAGAGCCGTGTTTGTCGCAGATACGCCGGTAGTCGTCCGTACACTGCGAATGCACAGACGCACTACCCAATGCGCCGCTAAGCGTAGTAACCACG

3. CAAGCGCCTCTGACGACCAACGGCTAGGAGCAGTAAAAGTGGCGGCAGTAGCAGTCGCAAACCTAGACCGAAGACGCCCGGGTGATGTGCCAGTTCGTCGAGTTTCTCTAGCTAGTTCTG

3' TGGTTCCCCGAAGTCGCGTTCCACTTCAGCCGGAAGCTCTTGAAGCAGCCCTGCGGCAACGTCGCCCACTTGCT GCACGCCGAGCGGCGGCAGTAGGACTACTAGGTCCAGCACGCCTGC

3. CCATCGGGCTAGTGCCGCAGGCTCTTCTGGACGTAGAAGCTCGAGTACCTTTTGGGCGACCTCTAAGTCTGCTAGAGCCGCTGGAAGCTCGCCCGCACCGCGATGTGCGTCCTCGCGGCO

31 GCCAAGAGCGAGCAGCGGCTGGGCGACGACGGCGACAAAGCGCTTTTCGACTGCCCGCTCCTCGCCTACGTCGAGCAGCTCTACGACTGGCTCCAGCGGAGGCGGGTAAGGCGGGCCTCO

3. CTCGACGTCGCTCTCCGCGACCGTGCCGACTTGGCAGCCGACTAGCGGCCGTTCGGCCTTAACCGCTTTTCGGCCCGGCCGGCGAAGCCGATCTTGCCTGGGGTGCTATTGCTTTTTCCG

3. GCGGGGCTTCCCCGCCGGGTGGCACCAGGGCGGCAGTCTCGCCCTIACAACAGCACGAAGAAGGTCCCGACGAGGTAGAGGAACCACGCGTCGTAGGGCTCGCGGGCGCGCTAGGCGACGC

3. CCACCTCACCCCGTACTAGTGGAGCAGCTACATCGGCGCGAGGCGGCGGTCCCTCCCGCTCTCCTTCGCCAGGAGCATAAGAAACCAGGCGAGCCGCTAGAACAGGCGTAGCGGCTAGAG T S H

3. CCGCGCCTTCTACTAGAGCTGTCGCGGGAACCGCGGGTAGTGGCGCTAGACGCGGCAGCCGGTGCGCATCAAGTCCAGCCGCGGCTGTACGAACCTCCGGTACTGCAGCATCCGCGGCGG

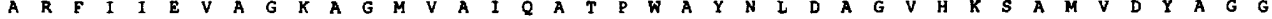

3. CTTCCGGAACGCCCACTACTACCAGTGCTCGCCGTGCCACCGAACCGACATCCGCTTCTCGTCGAACCGCGGCACGAACTAGTCCGGCGGTATAAGGACGCGGCACGGGCCGTCCTTCGG

GCCGTGCAGGTGCTTCCACTGCTAGCCCTAGCTCTTCCGCAACGTCTTCGCGTGCTTCGCCCGGCGGAAGGCCCTCCGCAGGCTTAGTTCGTGCGGCCGGTCGTGGTCGCCGACCAACCG P V D V F T V I P I S F A N C F R V F R A A K R S A D S D L V G A I V L P Q N A

3. CTGCTTTGGCTGCCACGCCGGGAGCTGCGCCGGCTTCGGCCACTATTATAACGCCCCGCATTTGGCGGACCTAGAGCTTCTTCAGCGGAAGCAGGTGCTGGGCCGCCTAGTCGAGCGGGTA $V F$ V T R G E R G F G I I R

3. CAGCATCCCGAACAAGCCCAACAGGCCCTAGTCGCACAGCTCGCTGAACAGCTCCGGCCAACTGCAGCAGCTTCGAGAGCTAGAGGCCGTGCGGGAGCTACAACAAGCGCCCGTCCTTCAG $\begin{array}{llllllllllllllllllllllllllllllllllllllll}D & Y & P & K & N & P & N & D & P & I & L & T & D & L & S & K & D & L & R & N & V & D & D & F & S & E & I & E & P & V & G & E & I & N & N & A & P & L & F & D\end{array}$

3. CTCGTCCGCCGCCTAGACCTCCTAGCGGAGCTGCAGCAAGAGCTTGCTTGGCAGCCGCTAGCTCCTAAAGCTCCACACCTGGAACCGCGGCGGCTCAAGGAGCCGCCAGTGCTGGAGCAM 8400

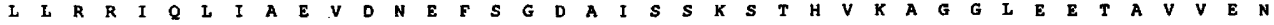

31. CCACTGCCAGAAGTGGTGCAGCCCCGGCCAGTGCTTGTACATCGAGCACAGTGCGTGGTACTTCTACTTCAGCCAGTACCGGCCGCTCATGTGCAGCGGCGGGCGCGTGCCGGGGTACTA T V T K V V D D P G T V T F M Y S S T

3. CTGGCTCTAGACGCCCTAGTGCGGCCTCCGTCGCTGCAACGCCGCCTTGTGGAGCGGCATCGGCGGCTCGCGCCGGTGCGGGAGGACCTACGCGCGTGGCGGCCGTAGCTTCTACGGCTA 8640

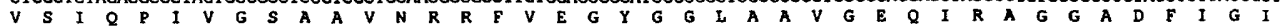

3. CTAGCCCCGCGCGTAGAAGTCGCGGTACAGGACCTGGAATTGCTAGAACCAACACACGCGGAGCCTCTCGCTCGOCGGCTTGTGGCACTTCAGGAAGCTCTTGTCCTTCTGCCACGCGGG

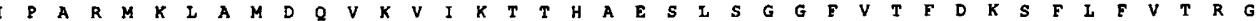

3. CAAGTGCCACGGGGTCGGCCACTGCTGCGGCGGCGGCCCCTAGAAGACGAAGAGGTACGGCTTTAGCCGCCTCGCCACGACGTGCTTGTACAGCTTGAGGAGCTTGCTGGGCACCAGCTC B8BO N V V $T$ T $G$ G W

3' GTCCTCAAGCTACGCGAGCGCGCGCCACTCGAACGGCGCGAACACGACGCGGAGCTCGGCGAAAAGGGGCGGCGGCTCTGCCCGGACACGCGCT GCGAGGAGTTCGA

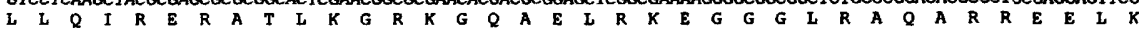

Fig. 2. Nucleotide and translated amino acid sequences of the 285 bp HindllI-EcoRI fragment (a) and 8988 bp EcoRI-HindIII fragment (b) of the $M$. extorquens AM1 chromosome region surrounding meaA. The coding DNA strands are shown. Hairpins are underlined and stop codons are indicated by asterisks. 


\section{Construction of insertion mutations}

Insertion mutations were generated in all the ORFs identified in the region under study. The strategy for introduction of specific mutations was based on the exchange of DNA via homologous recombination in vivo between the wild-type gene and a gene that had been inactivated by an insertion mutation in vitro (Ruvkun \& Ausubel, 1981). The $1.3 \mathrm{~kb} \mathrm{Km}^{\mathrm{r}}$ cassette originating from pUC4K (Vieira \& Messing, 1982) was employed, cut by appropriate restriction enzymes. The $\mathrm{Km}^{\mathrm{r}}$ cassette was always inserted in the orientation such that the direction of transcription of the $a p b$ gene coincided with the direction of transcription of the interrupted gene. This has been shown in the past to create non-polar insertions (Chistoserdova \& Lidstrom, 1994a, c; Chistoserdov et al., 1994). Plasmids in which corresponding genes had been interrupted by the insertion were then ligated with the suicide vector pAYC61 (Chistoserdov et al., 1994). The resulting plasmids were transformed into $E$. coli S17-1 to produce donors, which were mated with $M$. extorquens AM1. $\mathrm{Km}^{\mathrm{r}} M$. extorquens AM1 progeny were routinely obtained on plates containing succinate as the growth substrate, except in the case of mutations in $i l v C$, when supplements of valine and isoleucine $\left(1 \mathrm{mg} \mathrm{m}^{-1}\right.$ or $5 \mathrm{mg}$ $\mathrm{ml}^{-1}$, respectively) were also added. Colonies resistant to $\mathrm{Km}$ were checked for their resistance to $\mathrm{Tc}$. $\mathrm{Tc}^{\mathrm{s}}$ colonies were assumed to be double-crossover recombinants, while $\mathrm{Tc}^{\mathrm{r}}$ colonies were assumed to be singlecrossover recombinants. In all cases, the identity of the mutants was confirmed by hybridization of chromosomal DNA with corresponding specific probes and pUC4K DNA in comparison with the wild-type DNA (data not shown). Specific sites of insertions into the region are as follows (noted by asterisks in Fig. 1). Mutations 6211 and $6112 \mathrm{KH}$ are, approximately in the middle (EcoRI site) and in the end (Tth111I site) of $x d h$, respectively. Mutation $61 \mathrm{EP} 2$ is a $S p h \mathrm{I}$ deletion of approximately $0.3 \mathrm{~kb}$ involving the $5^{\prime}$ terminus of orf 1 . Mutation $61 \mathrm{MCM}$ is in the $S m a$ site approximately in the middle of mea $A$. Mutation $6128 \mathrm{Km}$ is in kat $A$ (PstI site at position 939, Fig. $2 \mathrm{~b}$ ). Mutation 61 ADH is in adh $A$ (BsaAI site at position 1684). Mutation 6132 is in the region separating $a d h A$ and orf 2 ( $N r u$ I deletion of 389 bp, positions 3287 and 3676, Fig. 2b). Mutation 61OR2 is in orf2 (EcoRI site at position 4507). Mutation 61EPKm and 61ICP are in ilvC (AvaI deletion of $190 \mathrm{bp}$, positions 5076 and 5266, and insertion into Pst I, position 5488 , respectively). Mutation $6111 \mathrm{H}$ is in the region separating $i l v C$ and $p c c B$ (BsaAI site at position 6797). Mutation 61PCC is in $p c c B$ (StuI site at position 7805).

\section{Phenotypic analysis of insertion mutants}

Up to 300 recombinants from each mating were tested for resistance to $\mathrm{Tc}$ in order to separate single-crossover $\left(\mathrm{Tc}^{\mathrm{r}} \mathrm{Km}^{\mathrm{r}}\right)$ and double-crossover $\left(\mathrm{Tc}^{\mathrm{s}} \mathrm{Km}^{\mathrm{r}}\right)$ recombinants. Mutations in only four genes produced double-crossover recombinants (with a frequency of $10-50 \%$ in relation to the total number of recombinants): mea $A, \operatorname{kat} A, a d h A$ and $\operatorname{pcc} A$ (Fig. 1). One of the insertions introduced into an intergenic site (mutation 6132) also resulted in double- crossover recombinants. Mutations in the other seven sites, including one mutation in an intergenic site $(6111 \mathrm{H})$ produced only single-crossover recombinants. Since those types of crossover events regenerate a functional target gene, this implies that these gene products are vital for growth of $M$. extorquens AM1 on succinate. It is not clear why mutation $6111 \mathrm{H}$ did not result in double-crossover recombinants, since past experience with insertions of the $\mathrm{Km}^{\mathrm{r}}$ cassette in $M$. extorquens AM1 did not cause polar effects (Chistoserdova \& Lidstrom, 1994a-c; Chistoserdov et al., 1994). All single-crossover mutants were able to grow on both $C_{1}$ and $C_{2}$ compounds (up to 300 colonies tested for each mutation event).

Growth responses have been determined for doublecrossover recombinant insertion mutants in mea $A$, adh $A$, kat $A$ and $p c c A$. Mutants in kat $A$ grew normally on $C_{1}$ (methanol and methylamine) and $\mathrm{C}_{2}$ (ethanol and ethylamine) compounds, while mutants in adh $A$, mea $A$ and $\operatorname{pcc} A$ lost the ability to grow on $\mathrm{C}_{1}$ and $\mathrm{C}_{2}$ compounds, and also on $\beta$-hydroxybutyrate, indicating that their products are required for both $\mathrm{C}_{1}$ and $\mathrm{C}_{2}$ metabolism. All mutants grew normally on pyruvate. Glyoxylate and glycolate have been used previously as supplements to characterize mutants in the unknown pathway of oxidation of acetyl-CoA to glyoxylate (Dunstan et al., 1972a, b; Dunstan \& Anthony, 1973; Salem \& Quayle, 1971; Salem et al., 1973). These compounds were tested for the ability to restore growth of the mutants on methanol, formate, ethylamine and $\beta$-hydroxybutyrate. Both supplements were able to restore growth of the mutants on $\mathrm{C}_{1}$ and $\mathrm{C}_{2}$ compounds and on $\beta$-hydroxybutyrate. Mutants in mea $A$ and adh $A$ regained the ability to grow on $C_{1}$ and $C_{2}$ compounds when the $13.5 \mathrm{~kb}$ HindIII fragment was introduced in a broad-hostrange vector, pRK310 (Ditta et al., 1985), while mutants in $p c c A$ were complemented by an overlapping $5.6 \mathrm{~kb}$ Pst I fragment isolated by hybridization and cloned into pRK310. This PstI fragment must therefore contain the complete $\operatorname{pcc} A$.

\section{Activity of catalase in katA mutants}

Activity of catalase was measured in a few representative kat $A$ mutants and in wild-type $M$. extorquens AM1 grown on methanol and succinate. In both the wild-type $M$. extorquens AM1 and the mutants, high levels of catalase activity were found, which in general seemed to be higher on succinate than on $C_{1}$ compounds (approximately 100 and $60 \mathrm{nmol} \mathrm{min}{ }^{-1}$ (mg protein) ${ }^{-1}$, respectively). These data suggested that $M$. extorquens AM1 may have multiple genes responsible for the synthesis of catalase. To prove the identity of the mutation in kat $A$, we separated proteins on isoelectrofocusing gels using crude extracts from the wild-type cells of $M$. extorquens AM1, from two representatives of kat $A$ mutants, CAT38 and CAT42, and from CAT38 carrying a plasmid containing cloned kat $A$ (pLC310.SS) and observed catalase activity by immersing the gels into a hydrogen peroxide solution. Two activity bands with $\mathrm{pI}$ values of approximately 4.6 and 5.0 were present in the wild-type extract, and also in the extract of CAT38(pLC310.SS), while only one (the less acidic) band 
Table 1. Activities of PCC, MCM and HPR in wild-type $M$. extorquens AM1, pccA and meaA insertion mutants, and in mutants carrying cloned pccA and meaA

Measurements were done in triplicate and values were within $\pm 25 \%$ of each other.

\begin{tabular}{|c|c|c|c|c|c|c|}
\hline \multirow[t]{3}{*}{ Strain } & \multicolumn{6}{|c|}{ Activity $\left[\mathrm{nmol} \mathrm{min}^{-1}(\mathrm{mg} \text { protein })^{-1}\right]$} \\
\hline & \multicolumn{2}{|c|}{ PCC } & \multicolumn{2}{|c|}{$\mathbf{M C M}$} & \multicolumn{2}{|c|}{ HPR } \\
\hline & Succ* & $\mathrm{MeOI}$ & Succ* & $\mathrm{MeO}$ & † Succ* & $\mathrm{MeOH} \dagger$ \\
\hline Wild-type & 25 & 20 & - & - & 380 & 1990 \\
\hline Wild-type (pLC310.84) & 65 & 40 & 15 & 10 & 360 & 1880 \\
\hline PCC50 & 0 & 0 & - & - & 360 & 1730 \\
\hline PCC54 & 0 & 0 & - & - & 350 & 1600 \\
\hline PCC50(pLC310.84) & 55 & 35 & 15 & 8 & 350 & 1900 \\
\hline MCM1(pLC310.84) & 50 & 30 & 15 & 10 & 400 & 1600 \\
\hline MCM28(pLC310.84) & 45 & 25 & 12 & 6 & 380 & 1580 \\
\hline MCM28(pLC310.SS84) & 60 & 30 & 18 & 9 & 350 & 1500 \\
\hline MCM28(pLC310.SS84) & 55 & 30 & 16 & 7 & 385 & 1775 \\
\hline
\end{tabular}

- , Not measured.

* Cells grown on succinate.

†Cells grown on methanol or, in the case of methanol-negative strains, grown on succinate, washed and incubated with methanol as described by Dunstan et al. (1972).

was present in the kat $A$ mutants (data not shown). Although the calculated $\mathrm{pI}$ for the product of kat $A$ is $6 \cdot 1$, the corresponding activity band shows a $\mathrm{pI}$ of approximately $4 \cdot 6$. This difference could be due to the quarternary structure of catalase, which is usually composed of four subunits (Murthy et al., 1981).

\section{Activity of PCC in PccA mutants}

PCC activity was measured in the wild-type $M$. extorquens AM1 cells and in two representatives of $p c c A$ mutants, PCC50 and PCC54. Cells from succinate-grown cultures or cultures grown on succinate and then induced with methanol (Dunstan et al., 1972b) were employed. The activity of HPR has also been used as a positive control for induction of serine cycle enzymes by methanol. Similar levels of PCC were found in wild-type $M$. extorquens AM1 grown on methanol or succinate. PCC activity was not found in $p c c A$ mutants. When a plasmid carrying cloned $p c c A$ (pLC310.84) was introduced into the $p c c A$ mutants, PCC activity was found at increased levels, in accordance with the presence of multiple copies of the gene in these strains (Table 1).

\section{Activity of MCM in meaA mutants}

Since mea $A$ appeared to be similar to genes encoding subunits of MCM, the presence of MCM was first checked in the mea $A$ mutants and in the wild-type cells. Since the assay we used involved PCC as a coupling enzyme, the latter was overexpressed by the introduction of plasmids carrying $p c c A$ (pLC310.84 carrying the $6.5 \mathrm{~kb}$ Pst I frag- ment containing entire $p c c A$ cloned into pRK310) or both mea $A$ and $p c c A$ (pLC310.SS84 carrying the $18.5 \mathrm{~kb}$ HindIII-PstI fragment). The activity was found at similar levels in mutant and wild-type cells grown on succinate or induced with methanol, and it did not seem to be overexpressed in strains carrying plasmids with cloned mea $A$ ('Table 1).

\section{Search for ADH activity}

The specificity of the product of $a d h A$ is unknown, but the enzyme reveals highest similarity with ADHs having specificity for short-chain alcohols (Russell et al., 1983; Russell \& Hall, 1983; Young \& Pilgrim, 1985; Gwynne et al., 1987; Saliola et al., 1990), which are most active on ethanol. ADHs are usually readily stained in gels (Megnet, 1967). We isoelectrofocused cell-free extracts of the wildtype $M$. extorquens AM1, two representatives of adh $A$ mutants, $\mathrm{ADH} 70$ and $\mathrm{ADH} 76$, and $\mathrm{ADH} 70$ carrying pLC310.SS (the $13.5 \mathrm{~kb}$ HindIII fragment cloned into pRK310), in which the adh $A$ product should be overexpressed. The gels were stained in various conditions, employing the following alcohols and aldehydes: ethanol, 2-propanol, glycerol, acetaldehyde, propionaldehyde, glycolaldehyde, glycolate and glycerate. As many as ten specific bands could be detected using the substrates above, and at least two of these seemed to be induced by $\mathrm{C}_{1}$ substrates (with $\mathrm{pI}$ values of approximately 6.5 and 7.5). However, none of the bands disappeared in the $a d h A$ mutants (data not shown). Thus, the identity of $a d b A$ remains unknown. 


\section{DISCUSSION}

In most aerobic organisms able to utilize acetate, the main assimilatory mechanism is the glyoxylate cycle, which is characterized by the presence of two enzymes: isocitrate lyase and malate synthase, in addition to enzymes of the tricarboxylic acid cycle. This pathway has been found in many bacteria, including $\mathrm{Icl}^{+}$serine methylotrophs, fungi, algae and protozoa (Gottschalk, 1979; Dawes \& Sutherland, 1994). $\mathrm{Icl}^{-}$serine methylotrophs, exemplified by $M$. extorquens AM1, lack isocitrate lyase, and the pathway used for assimilation of acetate, which also operates during growth on $\mathrm{C}_{1}$ compounds and $\beta$ hydroxybutyrate, remains unknown. It has been shown previously using ${ }^{14} \mathrm{C}$-labelled $\mathrm{C}_{1}$ and $\mathrm{C}_{2}$ compounds that glycolate, glyoxylate, malate and an unidentified compound (compound $\mathrm{X}$ ) must be intermediates in this unknown pathway (Dunstan et al., 1972a, b; Dunstan \& Anthony, 1973). These conclusions were confirmed by the isolation of mutants unable to utilize $C_{1}$ and $C_{2}$ compounds and $\beta$-hydroxybutyrate, which were complemented by the addition of glycolate or glyoxylate (Dunstan et al., 1972a, b; Dunstan \& Anthony, 1973; Salem \& Quayle, 1971; Salem et al., 1973). However, a pathway that converts acetyl-CoA to glyoxylate in this strain has never been identified. In this study we have approached the problem using molecular genetic analysis. A fragment of the $M$. extorquens AM1 chromosome previously used to complement such a mutant, PCT48, was sequenced in order to identify the gene defective in this mutant and possibly other genes involved in the pathway. Three genes specifically involved in the unknown pathway have been identified, the one defective in PCT48 (mea $A$ ) encoding a coenzyme $\mathrm{B}_{12}$-linked mutase and two new genes ( $p c c A$ and $a d h A$ ) encoding respectively PCC and an $\mathrm{ADH}$ with an as yet unknown function. Mutants in mea $A, \operatorname{pcc} A$ and $a d h A$ have the expected phenotype, i.e. they are $\mathrm{C}_{1}$ - and $\mathrm{C}_{2}$-negative, and are complemented on these substrates by glycolate or glyoxylate. These three genes, although found within a single DNA fragment, are obviously not co-transcribed and are separated from each other by non-coding DNA regions and by genes whose involvement in $C_{1}$ and $C_{2}$ metabolism we were not able to prove, since mutants in them were only obtained as single-crossover recombinants with $\mathrm{C}_{1}$ and $\mathrm{C}_{2}$-positive phenotypes. In addition to the newly identified genes apparently involved in the unknown part of the serine cycle, we have previously shown that serine hydroxymethyltransferase and the product of an unidentified gene (orf4) are probably involved in this pathway (Chistoserdova \& Lidstrom, 1994b, c). Phenotypes of mutants in $g l y A$ and orf 4 are different from the phenotypes of the mutants described here, since they were only complemented on $\mathrm{C}_{2}$ compounds by glyoxylate, and not by glycolate.

A metabolic pathway, the 3-hydroxypropionate cycle, has been described in which acetyl-CoA is converted into glyoxylate, which would involve PCC (Strauss \& Fuchs, 1993). In this pathway, acetyl-CoA is carboxylated and reductively converted via 3-hydroxypropionate to propionyl-CoA, which is carboxylated and converted via
succinyl-CoA to malyl-CoA. The latter is cleaved to acetyl-CoA and glyoxylate. A different pathway was proposed to operate in the serine methylotroph Protaminobacter ruber (Shimizu et al., 1984), in which glyoxylate could be regenerated from acetyl-CoA by using part of the tricarboxylic acid cycle to convert acetyl-CoA to $\alpha$ ketoglutarate. The latter is transformed to glutamate, followed by conversion of glutamate to mesaconate via $\beta$ methylaspartate, activation of mesaconate with CoA to form mesaconyl-CoA and cleavage of mesaconyl-CoA to glyoxylate and propionyl-CoA. The latter is then carboxylated to methylmalonyl-CoA and transformed to succinylCoA, which can be regenerated in another part of the tricarboxylic acid cycle to produce oxaloacetate, the acceptor for the next molecule of acetyl-CoA. None of the above pathways, however, would require any NADlinked $\mathrm{ADH}$, or a mutase other than MCM or serine hydroxymethyltransferase.

Two sequential reactions involving MCM and PCC are parts of both pathways described above. These are also shown to participate in $\beta$-oxidation of fatty acids and in degradation of branch-chained amino acids (Gottschalk, 1979). Both activities are present in $M$. extorquens AM1, but only PCC is proven to be specifically involved in the unknown pathway for regeneration of glyoxylate. mea $A$ apparently encodes a mutase different from MCM, and MCM is not able to substitute for its activity. The physiological substrate for the alternative mutase and consequently its role remain unknown.

\section{ACKNOWLEDGEMENTS}

We thank Pat Goodwin for supplying pSS48.11. This work was supported by a grant from the NIH (GM36294).

\section{REFERENCES}

Anthony, C. (1982). The Biochemistry of Methylotrophs. London: Academic Press.

Beers, R. F., Jr \& Seizer, W. (1952). A spectrophotometric method for measuring the breakdown of hydrogen peroxide by catalase. J Biol Chem 195, 133-140.

Bell, G. I., Najarian, R. C., Mullenbach, G. T. \& Hallewell, R. A. (1986). cDNA sequence coding for human kidney catalase. Nucleic Acids Res 14, 5561-5562.

Blomqvist, K., Nikkola, M., Lehtovaara, P., Suihko, M.-L., Airaksinen, U., Straby, K. B., Knowles, J. K. C. \& Penttila, M. E. (1993). Characterization of the genes of the 2,3-butanediol operons from Klebsiella terrigena and Enterobacter aerogenes. $J$ Bacteriol 175, 1392-1404.

Bol, D. K. \& Yasbin, R. E. (1991). The isolation, cloning and identification of a vegetative catalase gene from Bacillus subtilis. Gene 109, 31-37.

Chistoserdov, A. Y, Chistoserdova, L. V., Mclntire, W. S. \& Lidstrom, M. E. (1994). Genetic organization of the mau gene cluster in Methylobacterium extorquens AM1: complete nucleotide sequence and generation and characteristics of mau mutants. $J$ Bacteriol 176, 4052-4065.

Chistoserdova, L. V. \& Lidstrom, M. E. (1991). Purification and characterization of hydroxypyruvate reductase from the facultative methylotroph Metbylobacterium extorquens AM1. J Bacteriol 173, 7228-7232. 
Chistoserdova, L. \& Lidstrom, M. E. (1992). Cloning, mutagenesis, and physiological effect of a hydroxypyruvate reductase gene from Metbylobacterium extorquens AM1. J Bacteriol 174, 71-77.

Chistoserdova, L. V. \& Lidstrom, M. E. (1994a). Genetics of the serine cycle in Metbylobacterium extorquens AM1: identification of $\operatorname{sga} A$ and $m t d A$ and sequences of $\operatorname{sga} A, \operatorname{ppr} A$, and $m t d A$. $J$ Bacteriol 176, 1957-1968.

Chistoserdova, L. V. \& Lidstrom. M. E. (1994b). Genetics of the serine cycle in $M$. extorquens AM1 : cloning, sequence, mutation, and physiological effect of $g l y A$, the gene for serine hydroxymethyltransferase. J Bacteriol 176, 6759-6763.

Chistoserdova, L. V. \& Lidstrom M. E. (1994c). Genetics of the serine cycle in $M$. extorquens AM1: identification, sequence, and mutation of three new genes involved in $\mathrm{C}_{1}$ assimilation, orf4, $m t k A$, and $m t k B$. $J$ Bacteriol 176, 7398-7404.

Daniels, D. L., Plunket, G., III, Burland, V. D. \& Blattner F. R. (1992). Analysis of the Escherichia coli genome - DNA sequence of the region from 84.5 to 86.5 minutes. Science 257, 771-778.

Dawes, I. W. \& Sutherland, I. W. (1994). Microbial Pbysiology, 2nd edn. Oxford: Blackwell Scientific.

Ditta, G., Schmidhauser, T., Yakobson, F., Lu, P., Liang, X., Finlay, D., Guiney, D. \& Helinski, D. (1985). Plasmids related to the broad host range vector, $p R K 290$, useful for gene cloning and monitoring gene expression. Plasmid 13, 149-153.

Dunstan, P. M. \& Anthony, C. (1973). Microbial metabolism of $C_{1}$ and $\mathrm{C}_{2}$ compounds. The role of acetate during growth of Pseudomonas AM1 on $C_{1}$ compounds, ethanol and $\beta$ hydroxybutyrate. Biochem $J$ 132, 797-801.

Dunstan, P. M., Anthony, C. \& Drabble, W. T. (1972a). Microbial metabolism of $C_{1}$ and $C_{2}$ compounds. The involvement of glycollate in the metabolism of ethanol and of acetate by Pseudomonas AM1. Biochem J 128, 99-106.

Dunstan, P. M., Anthony, C. \& Drabble, W. T. (1972b). Microbial metabolism of $C_{1}$ and $C_{2}$ compounds. The role of glyoxylate, glycollate and acetate in the growth of Pseudomonas AM1 on ethanol and on $C_{1}$ compounds. Biochem $J$ 128, 107-115.

Francalanci, F., Davis, N. K., Fuller, J. Q., Murfitt, D. \& Leadlay, P. F. (1986). The subunit structure of methylmalonyl-CoA mutase from Propionibacterium shermanii. Biochem J 236, 489-494.

Furuta, S., Hayashi, H., Hijikata, M., Miyazawa, S., Osumi, T. \& Hashimoto, T. (1986). Complete nucleotide sequence of cDNA and deduced amino acid sequence of rat liver catalase. Proc Natl Acad Sci US A 83, 313-317.

Godon, J.-J., Chopin, M.-C. \& Ehrlich, S. D. (1992). Branched-chain amino acid biosynthesis genes in Lactococcus lactis subsp. lactis. $J$ Bacteriol 174, 6580-6589.

Gottschalk, G. (1979). Bacterial Metabolism. Berlin: Springer-Verlag.

Gwynne, D. I., Buxton, F. P., Sibley, S., Davies, R. W., Lockington, R. A., Scazzocchio, C \& Sealy-Lewis, H. M. (1987). Comparison of the cis-acting control regions of two coordinately controlled genes involved in ethanol utilization in Aspergillus nidulans. Gene 51, 205-216.

Harder, W., Attwood, M. \& Quayle, J. R. (1973). Methanol assimilation by Hyphomicrobium spp. J Gen Microbiol 78, 155-163.

Heilmann, H. J., Magert, H. J. \& Gassen, H. G. (1988). Identification and isolation of glucose dehydrogenase genes of Bacillus megaterium M1286 and their expression in Escherichia coli. Eur J Biochem 174, 485-490.

Jansen, R., Kalousek, F., Fenton, W. A., Rosenberg, L. E. \& Ledley, F. D. (1989). Cloning of full-length methylmalonyl-CoA mutase from a cDNA library using the polymerase chain reaction. Genomics 4, 198-205.
Kalb, V. F. \& Bernlohr R. W. (1977). A new spectrophotometric assay for protein in cell extracts. Anal Biochem 82, 362-371.

Klein, B., Pawlovski, K., Horicke-Grandpierre, C., Schell, J. \& Topfer, R. (195): Isolation and characterization of a cDNA from Cuphea lanceolata encoding a $\beta$-ketoacyl-ACP reductase. Mol Gen Genet 233, 122-128.

Kraus J. P., Firgaira, F., Novotny, J., Kaloussek, F., Williams, K. R., Williamson, C., Ohura, T. \& Rosenberg, L. E. (1986). Coding sequence of the precursor of the rat propionyl-CoA carboxylase. Proc Natl Acad Sci. US A 83, 8049-8053.

Lamhonwah, A.-M., Barankiewicz, T. J., Willard, H. F., Mahuran, D. J., Quan, F. \& Gravel, R. A. (1986). Isolation of cDNA clones coding for the $\alpha$ and $\beta$ chains of human propionyl-CoA carboxylase: chromosomal assignments and DNA polymorphisms associated with $p c c A$ and $p c c B$ genes. Proc Natl Acad Sci US A 83, 4864-4868.

Lampel, K., Uratani, B., Chaudhry, G., Ramaley, R. \& Rudikoff, S. (1986). Characterization of the developmentally regulated Bacillus subtilis glucose dehydrogenase gene. J Bacteriol 166, 238-243.

Maniatis, T., Fritsch, E. F. \& Sambrook, J. (1982). Molecular Cloning: a Laboratory Manual. Cold Spring Harbor, NY: Cold Spring Harbor Laboratory.

Megnet, R. (1967). Mutants partially deficient in alcohol dehydrogenase in Schizosaccharomyces pombe. Arch Biocbem Biopbys 121, 194-201.

Meinkoth, J. \& Wahl, G. (1984). Hybridization of nucleic acids immobilized on solid supports. Anal Biochem 138, 267-284.

Murthy, M. R. N., Reid, T. J., III, Sicignao, A., Tanaka, N. \& Rossmann, M. G. (1981). Structure of beef liver catalase. J Mol Biol 152, 465-499.

Orr, E. C., Bewley, G. C. \& Orr, W. C. (1990). cDNA and deduced amino acid sequence of Drosophila catalase. Nucleic Acids Res 18, 3663.

Peel, D. \& Quayle, J. R. (1961). Microbial growth on $C_{1}$ compounds: isolation and characterization of Pseudomonas AM1. Biochem J 81, 465-469.

Peoples, O. P. \& Sinskey, A. J. (1989). Fine structure analysis of the Zoogloea ramigera $p b b A-p b b B$ locus encoding $\beta$-ketothiolase and acetoacetyl-CoA reductase. Nucleotide sequence of pbbB. Mol Microbiol 3, 349-357.

Russell, D. W., Smith, M., Williamson, V. M. \& Young, E. T. (1983). Nucleotide sequence of the yeast alcohol dehydrogenase II gene. $J$ Biol Chem 258, 2674-2682

Russell, P. \& Hall, B. D. (1983). The primary structure of the alcohol dehydrogenase gene from the fission yeast Schizosaccharomyces pombe. $J$ Biol Chem 258, 143-149.

Ruvkun, G. B. \& Ausubel, J. R. (1981). A general method for sitedirected mutagenesis in procaryotes. Nature 289, 85-88.

Saito, H. \& Miura, K.-I. (1963). Preparation of transforming deoxyribonucleic acid by phenol treatment. Biochim Biophys Acta 72, 619-629.

Salem, A. R. \& Quayle, J. R. (1971). Mutants of Pseudomonas AM1 that require glycollate or glyoxylate for growth on methanol and ethanol. Biochem J 124, 74P.

Salem, A. R., Wagner, C., Hacking, A. J. \& Quayle, J. R. (1973). The metabolism of lactate and pyruvate by Pseudomonas AM1. $J$ Gen Microbiol 76, 375-388.

Saliola, M., Shuster, J. R. \& Falcone, C. (1990). The alcohol dehydrogenase system in the yeast, Kluyveromyces lactis. Yeast 6, 193-204.

Schroeder, W. A., Shelton, J. R., Shelton, J. B., Robberson, B., Apell, G., Fang, R. S. \& Bonaventura, J. (1982). The complete amino 
acid sequence of bovine liver catalase and the partial sequence of bovine erythrocyte catalase. Arch Biochem Biophys 214, 397-421.

Shimizu S., Ueda, S. \& Sato, K. (1984). Physiological role of vitamin $\mathrm{B}_{12}$ in a methanol-utilizing bacterium, Protaminobacter ruber. In: Microbial Growth on $C_{1}$ Compounds, pp. 113-117. Edited by R. L. Crawford \& R. S. Hanson. Washington, DC: American Society for Microbiology.

Simon, R., Priefer, U. \& Puhler, A. (1983). Vector plasmids for in vivo manipulations of Gram-negative bacteria. In: Molecular Genetics of the Bacteria-Plant Interactions, pp. 98-106. Edited by A. Puhler. Berlin: Springer-Verlag.

Slabas, A. R., Chase, D., Nishida, I., Murata, N., Sidebottom, C., Safford, R., Sheldon, P. S., Kekwick, R. G. O., Hardie, D. G. \& Mackintosh, R. W. (1992). Molecular cloning of higher plant 3oxoacyl (acyl carrier protein) reductase. Sequence identities with the nod $G$ gene product of the nitrogen-fixing soil bacterium Rhizobium meliloti. Biochem $J$ 283, 321-326.

Smith, L. M. \& Goodwin, P. M. (1992). Complementation analysis of mutants defective in the conversion of acetyl-CoA to glyoxylate in Metbylobacterium extorquens AM1. Abstracts of 7 th International Symposium on Microbial Growth on $C_{1}$ Compounds, B54. Warwick, England

Smith, L. M., Meijer, W. G., Dijkhuizen, L. \& Goodwin, P. M. (1995). A gene similar to methylmalonyl CoA mutase is required for the assimilation of $\mathrm{C}_{1}$ and $\mathrm{C}_{2}$ compounds in Methylobacterium extorquens AM1. Abstracts of 8th International Symposium on Microbial Growth on $C_{1}$ Compounds, p. 38. San Diego, USA.
Stone, S. \& Goodwin, P. M. (1989). Characterization and complementation of mutants of Metbylobacterium AM1 which are defective in C-1 assimilation. J Gen Microbiol 135, 227-235.

Strauss, G. \& Fuchs, G. (1993). Enzymes of a novel autotrophic $\mathrm{CO}_{2}$ fixation pathway in the phototrophic bacterium Chloroflexus aurantiacus, the 3-hydroxypropionate cycle. Eur J Biochem 215, 633-643.

Tsuji, K., Tsien, H. C., Hanson, R. S., DePalma, S. R., Scholtz, R. \& LaRoche, S. (1990). $16 \mathrm{~S}$ ribosomal RNA sequence analysis for determination of phylogenetic relationship among methylotrophs. $J$ Gen Microbiol 136, 1-10.

Vieira, J. \& Messing, J. (1982). The pUC plasmids, an M13mp7derived system for insertion mutagenesis and sequencing with synthetic universal primers. Gene 19, 259-268.

Whitaker, J. R. \& Granum, P. E. (1980). An absolute method for protein determination based on difference in absorbance at 2.35 and $280 \mathrm{~nm}$. Anal Biochem 109, 156-159.

Wilkemeyer, M. F., Crane, A. M. \& Ledley, F. D. (1990). Primary structure and activity of mouse methylmalonyl-CoA mutase. Biochem J 271, 449-455.

Young, E. T. \& Pilgrim, P. (1985). Isolation and DNA sequence of $a d b 3$, a nuclear gene encoding the mitochondrial isozyme of alcohol dehydrogenase in Saccharomyces cerevisiae. Mol Cell Biol 11, 3024-3034.

Received 23 October 1995; revised 19 January 1996; accepted 23 January 1996. 